\title{
SURVIE EMBRYONNAIRE DU SAUMON ATLANTIQUE (SALMO SALAR L.) DANS UN COURS D'EAU DU SUD DE SON AIRE DE RÉPARTITION, LA NIVELLE
}

\author{
J. DUMAS (1), M. OLAÏZOLA (1), L. BARRIERE (1)
}

(1) INRA, Station d'Hydrobiologie, UMR ECOBIOP, Ecologie Comportementale et Biologie des Populations de Poissons, Quartier Ibarron, 64310 Saint Pée sur Nivelle, France.

Reçu le 28 septembre 2006

Accepté le 6 mars 2007

Received September 28, 2006

Accepted March 6, 2007

\section{RÉSUMÉ}

L'évaluation de la survie du saumon Atlantique entre la ponte et l'émergence des alevins hors des graviers est réalisée dans le bassin de la Nivelle dans les domaines accessible (trois tronçons) et non accessible (trois tronçons) aux géniteurs. Pour ce faire, des capsules d'incubation de $12 \mathrm{~cm}^{3}$, contenant des œufs fraîchement fécondés, sont insérées dans des frayères artificielles et naturelles. Le débit et la température de l'eau de surface, l'oxygène dissous des eaux de surface et interstitielle et les caractéristiques granulométriques du substrat des frayères sont contrôlés pendant toute la durée de l'expérience. La survie, de 28,6 \% en moyenne dans l'ensemble des sites, est plus élevée dans les tronçons de l'amont (52,7 \% dans la Très Haute Nivelle et son affluent, le Lapitxuri) que dans les autres sites, dont ceux actuellement colonisés par l'espèce (21,5\%). II n'est pas observé de différence de survie entre les frayères artificielles et naturelles. Les mortalités interviennent essentiellement lors des épisodes de crues avant l'éclosion. Une corrélation positive forte existe entre la saturation en oxygène de l'eau interstitielle et la survie. Pour une saturation minimum en oxygène au-dessous de $62 \%$, la survie reste inférieure à $27 \%$. En revanche, la composition granulométrique du substrat ne semble pas agir directement sur la survie. Ce facteur n'étant pas corrélé avec l'oxygène dissous, ceci suggère que dans ce cours d'eau, le flux circulant dans les frayères ne provient pas uniquement de l'eau de surface, mais aussi de la nappe alluviale pauvre en oxygène dissous.

Mots-clés : Stades embryonnaires, frayère, survie, oxygène, eau courante, Salmo salar.

\section{EGG-TO-FRY SURVIVAL OF ATLANTIC SALMON (SALMO SALAR L.) IN A RIVER OF THE SOUTHERN EDGE OF ITS DISTRIBUTION AREA, THE NIVELLE}

\begin{abstract}
Survival of Atlantic salmon from egg deposition to fry emergence was estimated in the Nivelle River watershed in the zone colonized by spawners (three stretches) and the upstream uncolonized zone (three stretches). Twelve $\mathrm{cm}^{3}$ incubation capsules, which contained green eggs, were inserted into artificial and natural redds. Discharge and temperature of surface water, oxygen content of surface and intra-gravel water, and gravel composition of redd substratum were monitored all over the experiment period. Survival, $28.6 \%$ overall, was higher in upstream stretches $(52.7 \%$ in the Very Upper Nivelle and its tributary, the Lapitxuri) than in the others, among which, those of the colonized zone (21.5\%). There was no survival difference
\end{abstract}


between artificial and natural redds. Mortality mostly occurred during freshets before hatching. A strong positive correlation was observed between oxygen saturation of interstitial water and survival. For a minimum oxygen saturation $<62 \%$, survival was $<27 \%$. On the other hand, gravel composition did not seem to directly influence survival. This factor was not related to oxygen content, suggesting that, in this river, intra-gravel flow does not exclusively come from surface water, but also from low oxygen content alluvial groundwater. salar.

Key-words : Egg and free embryo, redd, survival, oxygen, running water, Salmo

\section{INTRODUCTION}

La survie embryonnaire du saumon Atlantique (Salmo salar L.) sous les graviers des frayères est l'une des phases les plus cruciales de son cycle vital. Elle intervient entre décembre et début avril à la limite sud de l'aire de répartition de l'espèce. Selon les cours d'eau, les taux moyen de survie entre les œufs déposés et les alevins émergents peuvent osciller de 2 à 35 \% (MACKENZIE et MORING, 1988 ; HELAND et DUMAS, 1994 ; PAUWELS et HAINES, 1994 ; ESTOURNES, 1998 ; DUMAS et DAROLLES, 1999). Ils demeurent du même ordre de grandeur pour la Truite de mer (Salmo trutta L.), comme l'observent RUBIN et GLIMSÄTER (1996). Les mortalités sont essentiellement dues au colmatage des interstices du substrat, qui réduisent les apports d'oxygène et l'évacuation des déchets métaboliques, tels l'ion ammonium $\left(\mathrm{NH}_{4}{ }^{+}\right)$, sa forme non ionisée la plus toxique, l'ammoniaque $\left(\mathrm{NH}_{3}\right)$, et à moindre égard, les nitrites $\left(\mathrm{NO}_{2}\right)$, ainsi qu'à la destruction des frayères lors des crues (BURKHALTER et KAYA, 1977 ; CHAPMAN, 1988 ; LISLE et LEWIS, 1992 ; CRISP, 1993, 1996 ; RUBIN et GLIMSÄTER, 1996 ; INGENDAHL, 2001 ; GUERRIN et DUMAS, 2001).

Peu de renseignements sur la survie des embryons de saumon Atlantique dans les cours d'eaux du sud de l'Europe sont actuellement disponibles (BARRACOU, 1994 ; ESTOURNES, 1998 ; DUMAS et DAROLLES, 1999 ; DUMAS et MARTY, 2006). Afin de mieux comprendre le fonctionnement d'une de ces populations modélisée par DUMAS et PROUZET (2003), des observations de la survie embryonnaire et des relations entre ce paramètre et la qualité du substrat, ainsi que celle de l'eau interstitielle des frayères, sont conduites en milieu naturel dans la Nivelle et trois de ses affluents de décembre 2000 à mars 2001. Des frayères naturelles et artificielles, mimant des frayères naturelles, sont équipées de capsules d'incubation (tubes cylindriques grillagés de quelques centimètres cubes) contenant un nombre connu d'œufs fraîchement fécondés issus d'une même ponte (DUMAS et MARTY, 2006). Cette méthode présente l'avantage de n'entraîner que de faibles modifications du substrat lors de la mise en place des capsules et d'être facile à mettre en œuvre. Toutefois, les alevins restant prisonniers du tube, elle ne permet pas l'évaluation du pourcentage d'alevins qui émergent effectivement comme le permettent des boîtes d'incubation-émergence (RUBIN, 1995 ; ESTOUNES, 1998 ; DUMAS et MARTY, 2006) ; la survie peut être bonne jusqu'à ce stade, mais certaines qualités de substrat peuvent empêcher les alevins d'émerger (RUBIN, 1998). Les capsules sont retirées en mars pour évaluer la survie au stade correspondant à celui des émergences. Les caractéristiques initiales et finales des frayères artificielles (structure granulométrique, indices de qualité de frayère) et les paramètres physico-chimiques de l'eau interstitielle (température et oxygène dissous) sont observés afin de rechercher leur incidence sur la survie.

\section{MATÉRIEL ET MÉTHODES}

\section{Sites d'étude}

Fleuve côtier du Pays Basque, la Nivelle prend sa source en Espagne. Après un parcours de 39 km, elle se jette dans le Golfe de Gascogne à St Jean de Luz (Figure 1). 
Son bassin versant de $238 \mathrm{~km}^{2}$ présente une grande variété géologique où dominent les formations marno-calcaires (flysch). II est essentiellement agro-pastoral avec des surfaces importantes de landes sur les reliefs (DUMAS et HAURY, 1995 ; DUMAS et PROUZET, 2003). Le débit annuel moyen en aval de la confluence avec l'affluent principal, le Lurgorrieta, est de $5 \mathrm{~m}^{3} \cdot \mathrm{s}^{-1}$ (période 1969-2006). Les saumons accèdent aux 18 premiers kilomètres du cours principal en eau douce, constitué de la Basse Nivelle (d'Ascain au barrage d'Olha, en amont de Saint Pée) et de la Haute Nivelle (d'Olha au barrage Urrutienea près de la frontière) et à 4,7 km du principal affluent, le Lurgorrieta. La Très Haute Nivelle et son affluent, le Lapitxuri, ainsi que le Lizarrieta, partie amont du Lurgorrieta, également englobés dans l'étude, sont des zones non accessibles aux géniteurs actuellement, du fait de barrages infranchissables.

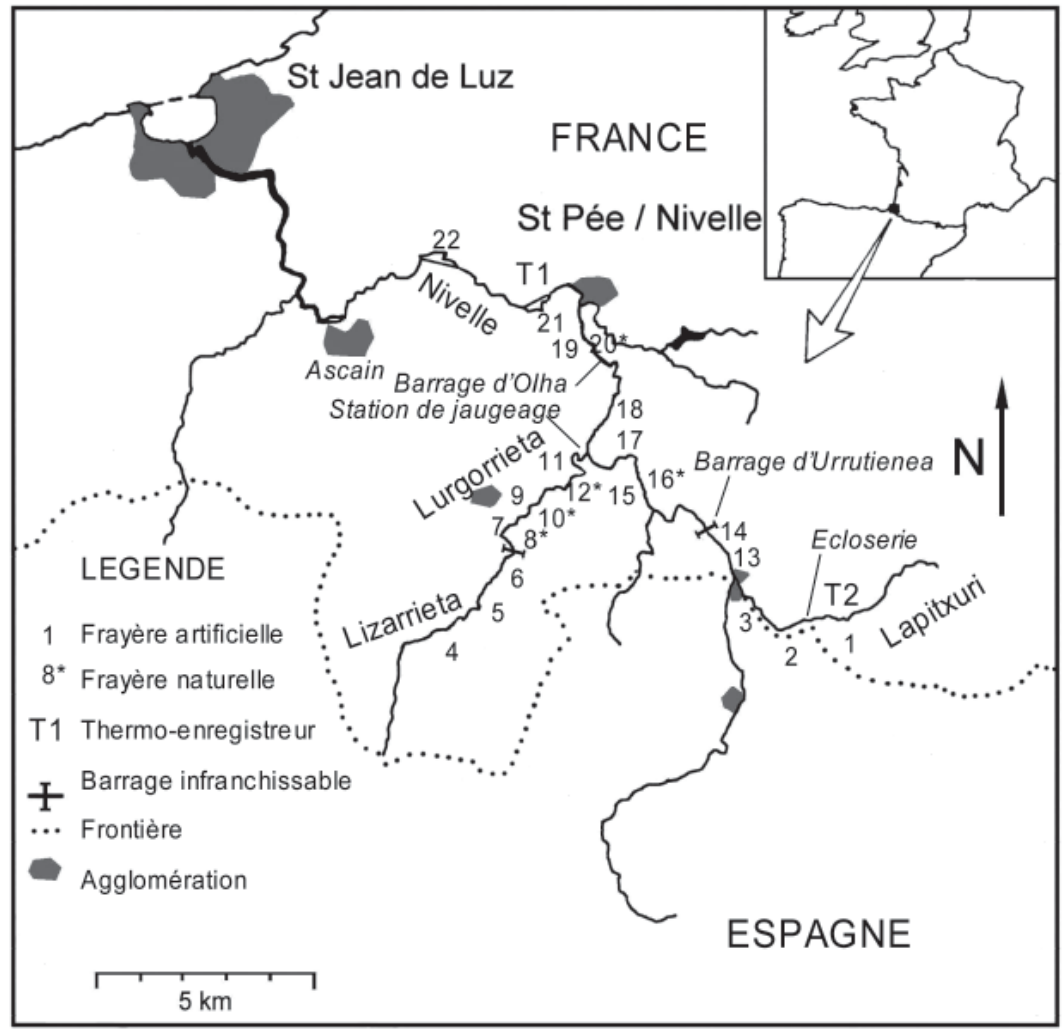

Figure 1

Le réseau hydrographique de la Nivelle. Localisation des frayères artificielles et naturelles, des thermomètres enregistreurs et de la station de jaugeage du débit.

Figure 1

The Nivelle River network. Localisation of the artificial and natural redd sites and of the temperature and discharge recorders.

\section{Localisation des frayères}

L'étude est effectuée dans 17 sites (Figure 1). Neuf sont localisés à des emplacements réguliers de frai naturel dans le domaine accessible aux migrateurs et 8 en domaine non colonisé, faisant l'objet d'un projet d'accessibilité, à des sites présentant des caractéristiques hydrodynamiques favorables au frai. A chacun de ces 
sites, une frayère artificielle est aménagée. Pour comparer la survie observée dans ces frayères artificielles avec celle des frayères naturelles, 5 frayères naturelles, voisines de frayères artificielles, sont également étudiées. Les 17 frayères artificielles se répartissent ainsi : trois dans la Basse Nivelle, trois dans la Haute Nivelle, trois dans le Lurgorrieta, deux dans la Très Haute Nivelle, trois dans le Lapitxuri et trois dans le Lizarrieta. Les 5 frayères naturelles sont localisées : une en Basse Nivelle, trois dans le Lurgorrieta et une en Haute Nivelle.

\section{Création et équipement des frayères (Figure 2)}

Les frayères sont créées et équipées du 8 au 18 décembre 2000. Elles sont positionnées en amont de radiers, comme le sont le plus souvent les frayères naturelles (BJORNN et REISER, 1991 ; DUMAS et DAROLLES, 1999). Les frayères artificielles sont réalisées par creusement au moyen d'une houe dentée en s'aidant du courant pour chasser les fines et en progressant vers l'amont dans l'axe du courant sur une distance d'environ $3 \mathrm{~m}$, le substrat remué remblayant la cavité en aval (DUMAS et MARTY, 2006). Une fois terminée, chaque frayère artificielle représente une tranchée de section trapézoïdale de $20 \mathrm{~cm}$ de large à la base et de $80 \mathrm{~cm}$ à la surface du substrat, recouverte de substrat remué sur $20 \mathrm{~cm}$ de profondeur, ce qui correspond à une profondeur normale
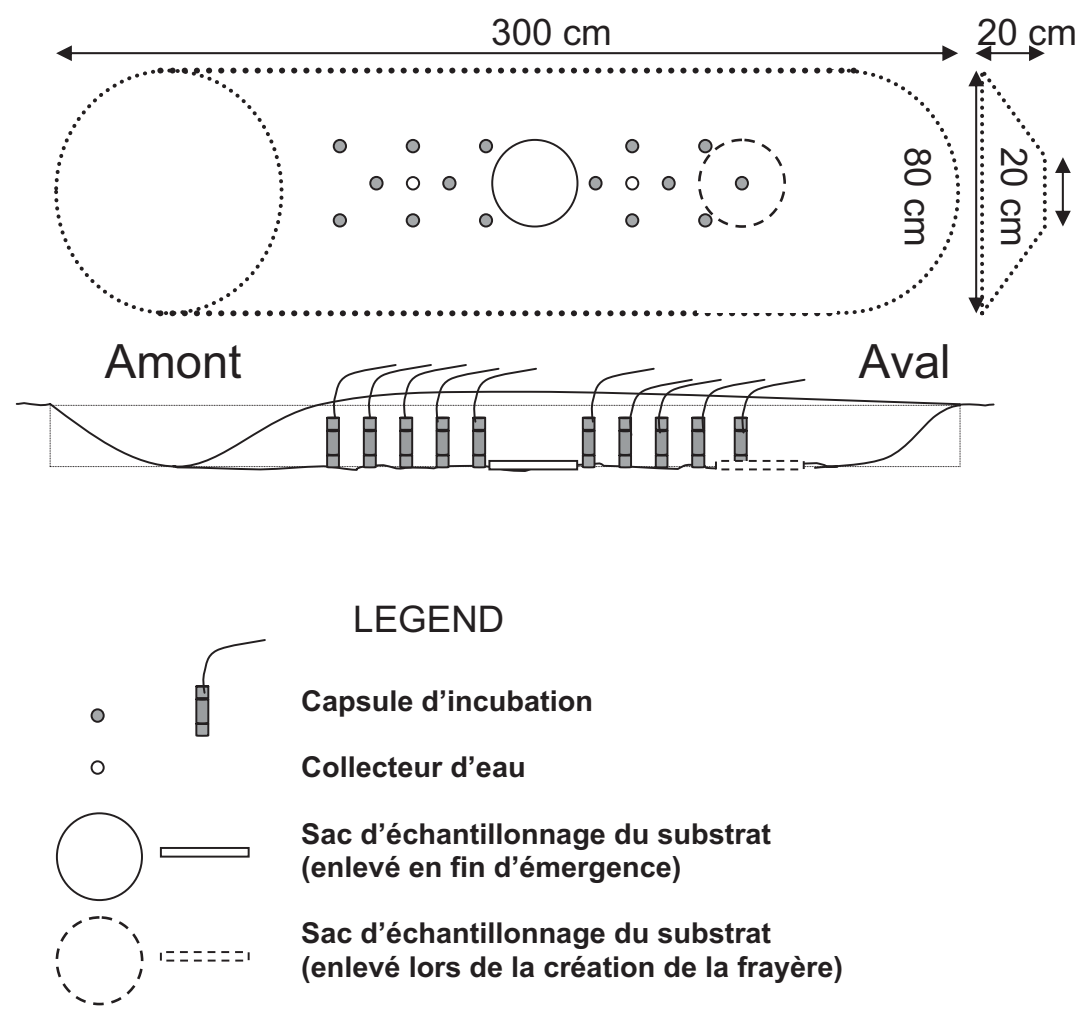

Figure 2

Schéma et dimensions des frayères artificielles et de leurs équipements : capsules d'incubation, sacs d'échantillonnage du substrat et collecteurs d'eau interstitielle (vue en plan et section longitudinale).

Figure 2

Schematic representation and measurements of artificial redds and the arrangement of their equipments: incubation capsules, substratum sampling bags and interstitial water collectors (ground-plan view and longitudinal section). 
d'enfouissement des œufs chez le saumon Atlantique (CRISP, 1996), avec une cavité en amont suivie d'un dôme allongé en aval.

II est prévu d'insérer 15 capsules formant deux groupes (8 et 7 capsules). A cet effet, chaque frayère est équipée de 15 tubes en PVC de $20 \mathrm{~cm}$ de long et de $1,7 \mathrm{~cm}$ de diamètre intérieur destinés à conduire les capsules dans le substrat. Ils sont placés au moyen d'une aiguille d'introduction (barre métallique pointue) sur laquelle le tube PVC est enfilé, et retenu par une collerette butoir. Une fois enfoncé dans le substrat à la profondeur voulue et la barre métallique retirée, le tube implanté est bouché pour éviter tout dépôt avant la mise en place des capsules d'incubation (DUMAS et MARTY, 2006). L'équipement est complété de deux sacs de prélèvement du substrat (l'un retiré dès que la frayère est terminée et l'autre y demeurant jusqu'à la fin de l'expérience) et de deux collecteurs d'eau interstitielle disposés chacun au centre d'un groupe de capsules (DUMAS et al., sous presse).

Les frayères naturelles ne sont pas équipées de sacs d'échantillonnage afin de ne pas perturber leur structure. Trois d'entre elles ne reçoivent qu'un seul collecteur d'eau.

\section{Caractéristiques morphométriques et granulométriques des frayères}

Chaque frayère est caractérisée par sa longueur, sa largeur au niveau du dôme et sa profondeur d'ambiance (moyenne de deux mesures prises de chaque côté du dôme), la profondeur du dôme, la profondeur de la dépression amont, la vitesse d'ambiance de la lame d'eau (moyenne de deux mesures prises aux mêmes emplacements que les profondeurs) et le régime d'écoulement, estimé par le nombre de Froude (DUMAS et DAROLLES, 1999).

Dans les frayères artificielles, un contrôle de l'évolution granulométrique du substrat durant l'incubation des œufs est effectué avec deux prélèvements de graviers, le premier dans le tiers aval, juste après la création de la frayère et le deuxième, au centre du dôme, à la fin des observations en mars. Ils sont réalisés à l'aide de sacs de prélèvement repliés, préalablement enfouis au niveau du substrat basal de la frayère, et permettent d'effectuer un carottage de la colonne de gravier (Figure 2). Chaque sac est doté d'une ouverture constituée d'une armature cerclée en aluminium d'un diamètre de $20 \mathrm{~cm}$, afin de donner une forme cylindrique à la poche. II est confectionné à partir d'un tissu très résistant, imputrescible et imperméable (d'une hauteur de $20 \mathrm{~cm}$ pour un volume utile d'environ 9 litres) fixé sur l'armature circulaire. II est pourvu de deux anses d'extraction en acier plastifié (17 cm de hauteur) positionnées verticalement et équipées chacune de cordons rouges de $50 \mathrm{~cm}$ de long dépassant de la surface des graviers afin de les localiser sur le fond de la rivière (DUMAS et al., sous presse).

Lors la création de la frayère, les sacs sont enfouis sous le remblai de gravier. Une fois celle-ci achevée, le premier sac est alors retiré pour vérifier l'état initial du substrat. La même manipulation est réalisée pour le deuxième sac lors du retrait des capsules en mars pour constater l'état final du substrat.

Le contenu des sacs, rapporté au laboratoire, est séché dans une étuve à $60^{\circ} \mathrm{C}$ pendant $12 \mathrm{~h}$. La fraction supérieure à $40 \mathrm{~mm}$ de diamètre est éliminée, la présence des gros éléments dans les échantillons étant aléatoire (BESCHTA et JACKSON, 1979 ; DUMAS et DAROLLES, 1999 ; INGENDAHL, 2001). Le tamisage est effectué à l'aide d'une colonne de tamis de mailles dégressives $(20,10,5,2,1,0,4,0,2,0,1,0,063 \mathrm{~mm})$ sur un tamiseur automatique Retsch AS 200 digit. La fraction retenue sur chacun d'eux et sur le récipient de fond est ensuite pesée. Les échantillons sont caractérisés par les proportions des différentes fractions granulométriques, le diamètre moyen géométrique (Dg), le coefficient de Kumbein et Pettijohn ou coefficient de tri (So) qui s'éloigne d'autant plus de la valeur 1 que son substrat est diversifié et l'indice de qualité du substrat pour l'incubation des salmonidés ou indice de fredle (Fi), dont la valeur augmente avec la qualité de la frayère (LOTSPEICH et EVEREST, 1981) utilisés par DUMAS et DAROLLES (1999), DUMAS et MARTY, (2006) et DUMAS et al., (sous presse). 


\section{Caractéristiques physico-chimiques des eaux de surface et interstitielles}

La température des cours d'eau est enregistrée avec des thermographes JRI (Jules Richard Instruments, Argenteuil, France) à Zaldubia (Saint Pée sur Nivelle, Figure 1) pour la Nivelle (T1) et à la pisciculture du Lapitxuri (T2). Le débit journalier moyen de la Nivelle est obtenu à la station de jaugeage de Cherchebruit, gérée par la DIREN Midi Pyrénées, $6 \mathrm{~km}$ en amont (Figure 1). Afin de le comparer avec ceux d'études précédentes sur le même sujet dans ce réseau hydrographique (DUMAS et DAROLLES, 1999 ; ESTOURNES, 1998 ; DUMAS et MARTY, 2006), il est recalculé pour le site de Zaldubia en tenant compte de l'augmentation de surface du bassin versant (facteur multiplicatif de 1,174).

La qualité de l'eau de surface et interstitielle des frayères est contrôlée à 7 dates au cours de la période d'expérimentation (le 21/12 en 2000 et les 5/01, 17/01, 2/02, 15/02, $26 / 02$ et 6/03 en 2001). Cette périodicité convient pour vérifier les tendances d'évolution à long terme des caractéristiques de l'eau, mais elle ne permet pas d'évaluer des changements de courtes durées, qui peuvent passer inaperçus. La température $\left({ }^{\circ} \mathrm{C}\right)$ et le taux d'oxygène (en mg. ${ }^{-1}$ et en \% de saturation) de l'eau de surface et interstitielle sont mesurés pour chaque site à l'aide d'un oxythermomètre de terrain WTW OXI 330.

L'eau de surface est analysée en introduisant la sonde dans la colonne d'eau située au-dessus des frayères. L'eau interstitielle est échantillonnée au moyen des collecteurs d'eau préalablement installés dans les frayères au même niveau que les capsules. De mêmes formes et dimensions que ces dernières, ils sont constitués de chevilles-tamis (petits cylindres en grillage de PVC moulé, de $9 \mathrm{~cm}$ de long et de $1,4 \mathrm{~cm}$ de diamètre) recouvertes d'un tissu synthétique à maille fine qui sont reliées à la surface par un tube de plastique souple et fin de $4 \mathrm{~mm}$ de diamètre extérieur permettant l'installation d'une seringue d'extraction de l'eau (DUMAS et MARTY, 2006). Un échantillon d'eau interstitielle est réalisé dans chaque groupe de capsules de chacune des frayères. Lors de l'échantillonnage, afin d'éliminer l'eau stagnant dans le préleveur, $20 \mathrm{ml}$ sont aspirés et rejetés avant d'extraire $100 \mathrm{ml}$ d'eau à analyser.

\section{Contrôle de la survie}

\section{Capsules d'incubation}

Les capsules d'incubation utilisées sont les mêmes que celles décrites par DUMAS et MARTY (2006). II s'agit de petits tubes cylindriques en grillage d'acier inoxydable de $1,5 \mathrm{~mm}$ de vide de maille, de $9 \mathrm{~cm}$ de longueur pour un diamètre de $1,4 \mathrm{~cm}$. Un bouchon ferme chacune des extrémités, ménageant un volume utile de $12 \mathrm{~cm}^{3}$. Un fil en nylon d'environ $50 \mathrm{~cm}$ est relié à l'un des bouchons collé à la capsule. Ce fil permet de localiser et de retirer les capsules en fin d'observations.

\section{Manipulation des œufs et insertion des capsules}

Une ponte artificielle est obtenue le 19 décembre 2000 en début de matinée à la pisciculture du Lapitxuri. Les géniteurs, une femelle et deux mâles d'un an de mer proviennent de la station de contrôle d'Uxondoa en Basse Nivelle. Après observation d'un temps de repos et de gonflement dans l'eau d'une demi-heure après la fécondation, des lots de 15 capsules contenant 10 œufs chacune, soit 150 œufs, destinés à équiper chacune des frayères, sont constitués et placés dans des glacières remplies d'eau pour éviter les chocs.

Un lot témoin de 200 œufs est conservé à l'écloserie de la pisciculture du Lapitxuri dans une armoire d'incubation Heath alimentée par l'eau filtrée du ruisseau. Ce lot subit des variations de température proches de celles subies par les autres lots en cours d'eau aux mêmes dates.

Les capsules sont introduites dans les frayères au moyen des tubes conducteurs préalablement insérés, lesquels sont retirés après l'implantation (DUMAS et MARTY, 
2006). Les œufs regroupés dans la partie inférieure des capsules se trouvent alors enfouis entre 14 et $18 \mathrm{~cm}$ sous la surface du substrat. L'insertion des capsules dans les frayères a lieu entre 3 et $9 \mathrm{~h}$ après la fécondation.

\section{Retrait des capsules et évaluation de la survie}

Les différents stades de développement du lot témoin sont observés à l'écloserie où la température de l'eau est enregistrée, ce qui permet de calculer les degrés-jours (dj) nécessaires pour atteindre chacun d'eux. Pour les embryons des capsules, les dates de ces mêmes stades sont estimées d'après les degrés-jours correspondants, observés sur le lot témoin, et la température des cours d'eau enregistrée à Saint Pée. Le 6 mars 2001, soit à 770 dj qui correspondent, dans le lot témoin, à la fin de de la résorption de la vésicule vitelline et au stade auquel les alevins émergent en milieu naturel, les capsules sont extraites des frayères et pour chacune d'elles les alevins vivants, les alevins morts et les œufs morts sont dénombrés.

\section{RÉSULTATS}

\section{Conditions générales d'incubation}

Au cours de la période de développement embryonnaire, du 19 décembre au 6 mars, les moyennes des températures sont de $9,9^{\circ} \mathrm{C}$ (écart-type $=1,4{ }^{\circ} \mathrm{C}$ ) dans la Nivelle et de $10,5^{\circ} \mathrm{C}$ (écart-type $=1,1^{\circ} \mathrm{C}$ ) dans le Lapitxuri. Les moyennes journalières fluctuent de $5,6^{\circ} \mathrm{C}$ le 28 février à $12,9^{\circ} \mathrm{C}$ le 6 mars (Figure 3a). Elles sont normales pour le réseau hydrographique de la Nivelle et compatibles avec les exigences de développement du saumon Atlantique.

Le débit moyen durant l'expérience est de $6,7 \mathrm{~m}^{3} \cdot \mathrm{s}^{-1}$ et 7 crues de moyenne importance (entre 11,5 et $15,6 \mathrm{~m}^{3} \cdot \mathrm{s}^{-1}$ ) caractérisent le régime hydrologique pendant l'expérience (Figure 3b). Deux des crues interviennent peu de temps avant l'embryonnement (stade auquel les yeux de l'embryon deviennent apparents à travers l'enveloppe de l'œuf), quatre autres avant et en début d'éclosion et la dernière peu avant la résorption complète des sacs vitellins du lot témoin en écloserie (correspondant à l'émergence; Figure 3a). Aucune de ces crues n'a d'effet sur les équipements des frayères.

\section{Caractéristiques morphométriques des frayères}

Les frayères artificielles, implantées sur des sites de frai dans le domaine accessible au saumon ou des sites potentiels dans le domaine inaccessible présentent des caractéristiques voisines de celles des frayères naturelles (Tableau I). Les vitesses

\section{Tableau I}

Caractéristiques morphologiques des frayères artificielles et naturelles de saumon Atlantique et de leur proche environnement.

\section{Table I}

Morphological characteristics of the artificial and natural redds of Atlantic salmon and of their surrounding environment.

\begin{tabular}{cccccccccc}
\hline Frayère & Nombre & $\begin{array}{c}\text { Longueur } \\
(\mathbf{c m})\end{array}$ & $\begin{array}{c}\text { Largeur } \\
\mathbf{( c m})\end{array}$ & $\begin{array}{c}\text { Profondeur } \\
\text { du fond } \\
\text { initial } \\
\mathbf{( c m}\end{array}$ & $\begin{array}{c}\text { Profondeur } \\
\text { du dôme } \\
(\mathbf{c m})\end{array}$ & $\begin{array}{c}\text { Profondeur de } \\
\text { la dépression } \\
\text { amont } \\
(\mathbf{c m})\end{array}$ & $\begin{array}{c}\text { Différence } \\
\text { dôme- } \\
\text { dépression } \\
\text { (cm) }\end{array}$ & $\begin{array}{c}\text { Vitesse } \\
\left(\mathbf{c m} . \mathbf{s}^{-1}\right)\end{array}$ & $\begin{array}{c}\text { Nombre } \\
\text { de Froude }\end{array}$ \\
\hline Artificielle & 17 & $294 \pm 24,3$ & $79 \pm 5,4$ & $30 \pm 6,3$ & $25 \pm 6,3$ & $52 \pm 8,5$ & $27 \pm 3,2$ & $57 \pm 14$ & $0,33 \pm 0,07$ \\
\hline Naturelle & 5 & $321 \pm 151$ & $102 \pm 15$ & $29 \pm 4,1$ & $25 \pm 6,4$ & $42 \pm 4,4$ & $17 \pm 3,5$ & $57 \pm 12$ & $0,34 \pm 0,05$ \\
\hline
\end{tabular}



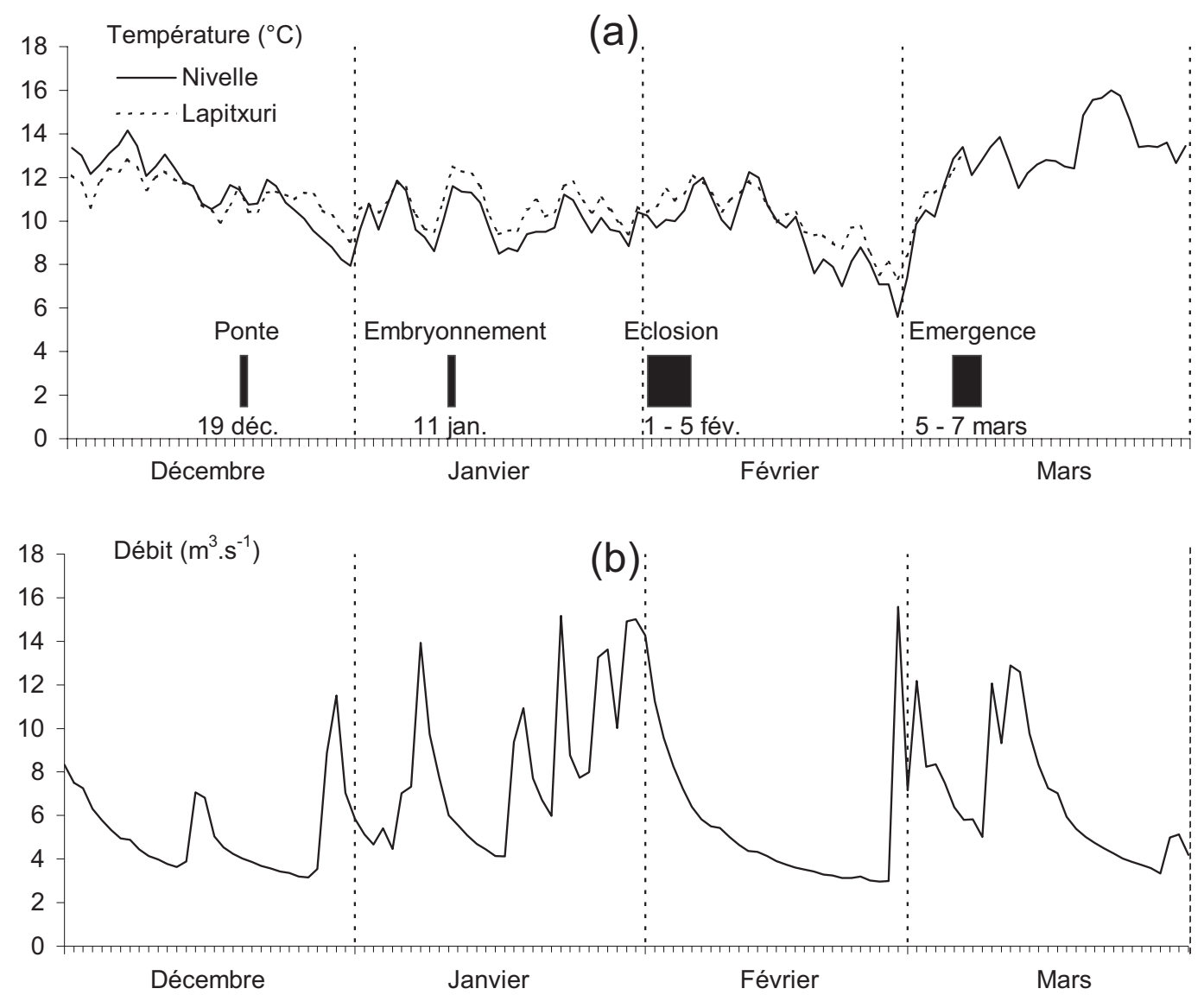

\section{Figure 3}

(a) Dates des différents stades de développement de la ponte artificielle de saumon Atlantique, températures moyennes journalières de la Nivelle et du Lapitxuri de décembre 2000 à mars 2001 ; (b) débit moyen journalier de la Nivelle à Zaldubia.

\section{Figure 3}

(a) Dates of the various development stages for the artificial spawn of Atlantic salmon, mean daily temperatures of the Nivelle at Zaldubia and of the Lapitxuri Brook from December 2000 to March 2001; (b) mean daily discharges of the Nivelle at Zaldubia.

moyennes d'ambiance sont identiques $\left(57 \mathrm{~cm}^{-1}\right)$. Les nombres de Froude sont très voisins ( 0,34 pour les naturelles et 0,33 pour les artificielles) et sont l'indice d'un écoulement peu perturbé et non déstabilisateur du fond. Cependant, les frayères naturelles sont plus larges (test de Mann-Whitney, $\mathrm{N}_{1}=5, \mathrm{~N}_{2}=17, \mathrm{p}<0,01$ ), présentent une dépression amont plus profonde (test de Mann-Whitney, $\mathrm{N}_{1}=5, \mathrm{~N}_{2}=17, \mathrm{p}<0,05$ ) et une différence dômedépression moins importante que les frayères artificielles (test de Mann-Whitney, $N_{1}=5$, $\left.\mathrm{N}_{2}=17, \mathrm{p}<0,001\right)$.

\section{Caractéristiques du substrat}

Les poids moyen des échantillons, $8,3 \mathrm{~kg}$ à la création des frayères et 7,6 kg à la fin de l'expérience, sont significativement différents (test t pour données appariées, $N=17$, $p<0,05)$. Ils reflètent ainsi un arasement de la couche supérieure du dôme, permettant de déduire sa perte de hauteur, évaluée à $1,7 \mathrm{~cm}$ en moyenne. 
Que ce soit aux états initiaux ou finals, plus de $50 \%$ du substrat sont constitués d'éléments $<10 \mathrm{~mm}$ et $17,6 \%$ ou plus de particules $<2 \mathrm{~mm}$ (Tableau II). Durant la période d'incubation, les fractions cumulées $<1 \mathrm{~mm}$ (8 et $9 \%$ en moyenne, respectivement en début et fin d'expérience) augmentent significativement de $12 \%$ ou plus selon les frayères par rapport à l'état initial (tests de Wilcoxon, $N=17,0,05>p>0,01$ ). Les fractions cumulées qui intègrent des éléments plus grossiers ne diffèrent pas significativement (Tableau II). L'hétérogénéité du substrat, traduite par So, augmente entre décembre et mars (différence presque significative : test de Wilcoxon, $\mathrm{N}=17, \mathrm{p}=0,06$ ). Les faibles diminutions de $\mathrm{Dg}$ et de l'indice de qualité du substrat de frai (Fi) ne sont pas significatives. Ces résultats vont dans le sens d'une légère dégradation du milieu d'incubation essentiellement due à l'accumulation des éléments les plus fins.

Toutes les comparaisons effectuées sur les fréquences et les indices granulométriques en fonction du domaine (accessible ou inaccessible) ou des ensembles de tronçons Lapitxuri-Très Hautes Nivelle, Lizarrieta-Lurgorrieta, Haute et Basse Nivelle, ne révèlent aucune tendance particulière (tests de Kruskal-Wallis, D.L. $=1$ dans le premier cas et D.L. $=2$ dans le second).

\section{Qualité de l'eau}

\section{Température}

Les températures de l'eau intra-gravier sont voisines ou identiques à celles de l'eau de surface. Elles sont du même ordre de grandeur que les températures de surface enregistrées aux thermographes de Zaldubia (Nivelle) et de la pisciculture pour les mêmes dates et heures.

\section{Oxygène dissous}

L'eau de surface, constamment brassée, est toujours saturée en oxygène.

En milieu interstitiel, il n'y a pas de différence de saturation moyenne en oxygène en fonction de la position amont $(78,3 \%)$ ou aval $(77,2 \%)$ dans la frayère (test t pour données appariées, $N=126$ ), hormis pour la frayère 6 en aval du Lizarrieta (85 \% en amont et $24 \%$ en aval).

La nature des frayères (artificielle ou naturelle) n'a pas d'influence sur le taux de saturation en oxygène aux cinq sites où les deux types de frayères sont placés en parallèle (76,1 \% dans les artificielles et $78,2 \%$ dans les naturelles ; test t pour données appariées, $\mathrm{N}=35)$.

Avec le temps, les concentrations moyennes de l'eau interstitielle varient de 10,6 à $6,9 \mathrm{mg}^{-1} \mathrm{I}^{-1}$ et la saturation de 98,1 à $63,3 \%$. Elles diffèrent beaucoup selon les frayères pour une même date (Figure $4 \mathrm{a})$. Après un chute rapide à $75,4 \%\left(8,1 \mathrm{mg}^{\left.-\mathrm{l}^{-1}\right)}\right.$ entre le $21 / 12 / 2000$ et le 5/01/2001, la saturation moyenne fluctue entre 69 et $76,1 \%$ (7,5 et $8,6 \mathrm{mg.l}^{-1}$ ) pour la période embryonnement-éclosion. Dans l'ensemble, on observe une diminution de près de $35 \%$ du début de l'incubation jusqu'à l'émergence. L'influence du facteur temps est très hautement significative (analyse de variance à un facteur, $F_{6,153}$, $\mathrm{p}<0,001)$.

Dans les conditions de cette expérience, la composition du substrat et les indices granulométriques aux états initiaux et finals ne semblent pas influencer la saturation en oxygène de l'eau interstitielle (corrélations de rang de Spearman non significatives, $\mathrm{N}=17)$.

II n'apparaît pas de différence significative entre les domaines accessible et inaccessible sur la saturation en oxygène $(75,3$ et $79,7 \%$ respectivement pour chacun ; test $t, N_{1}=98, N_{2}=56$ ). 


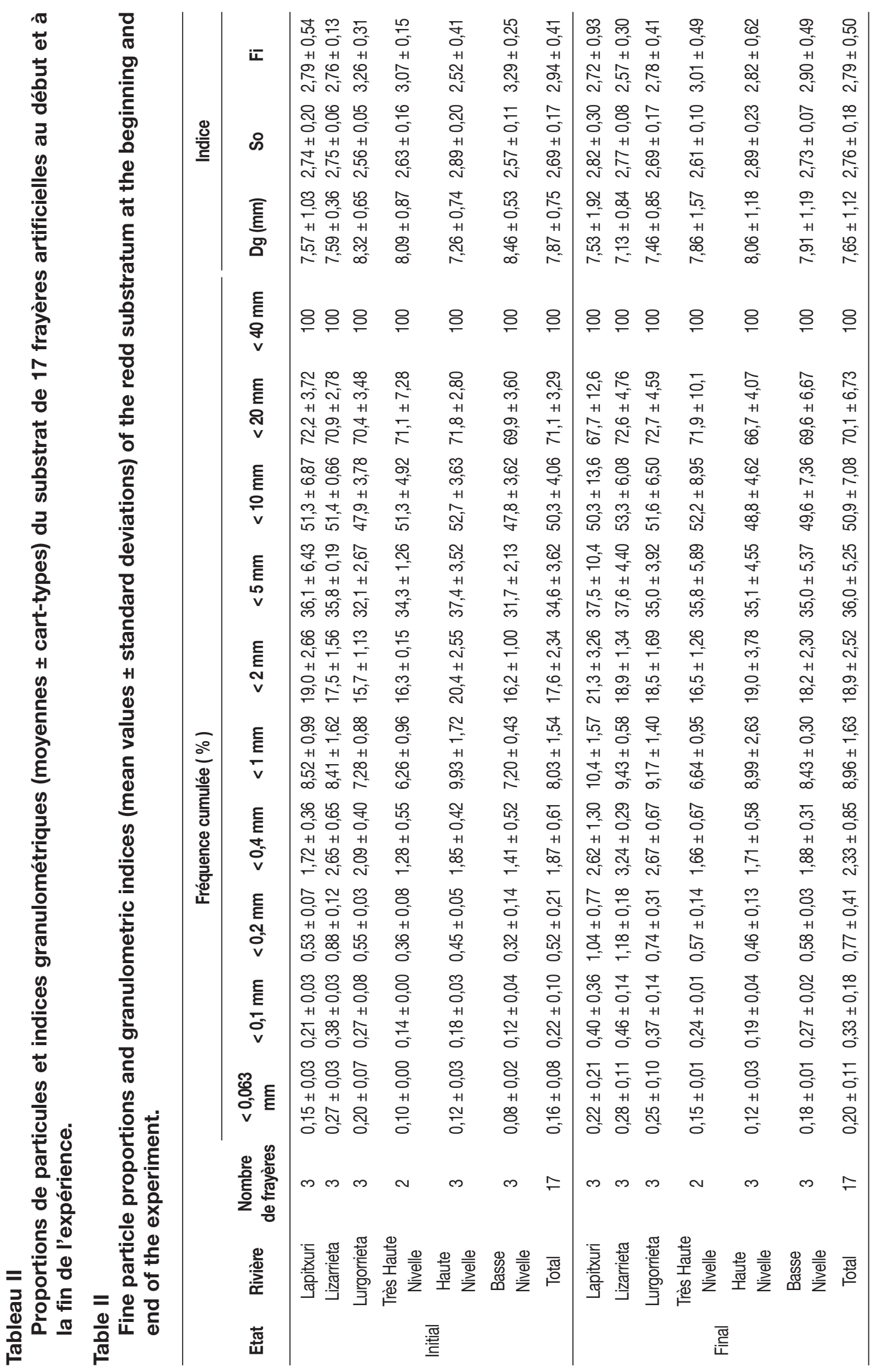



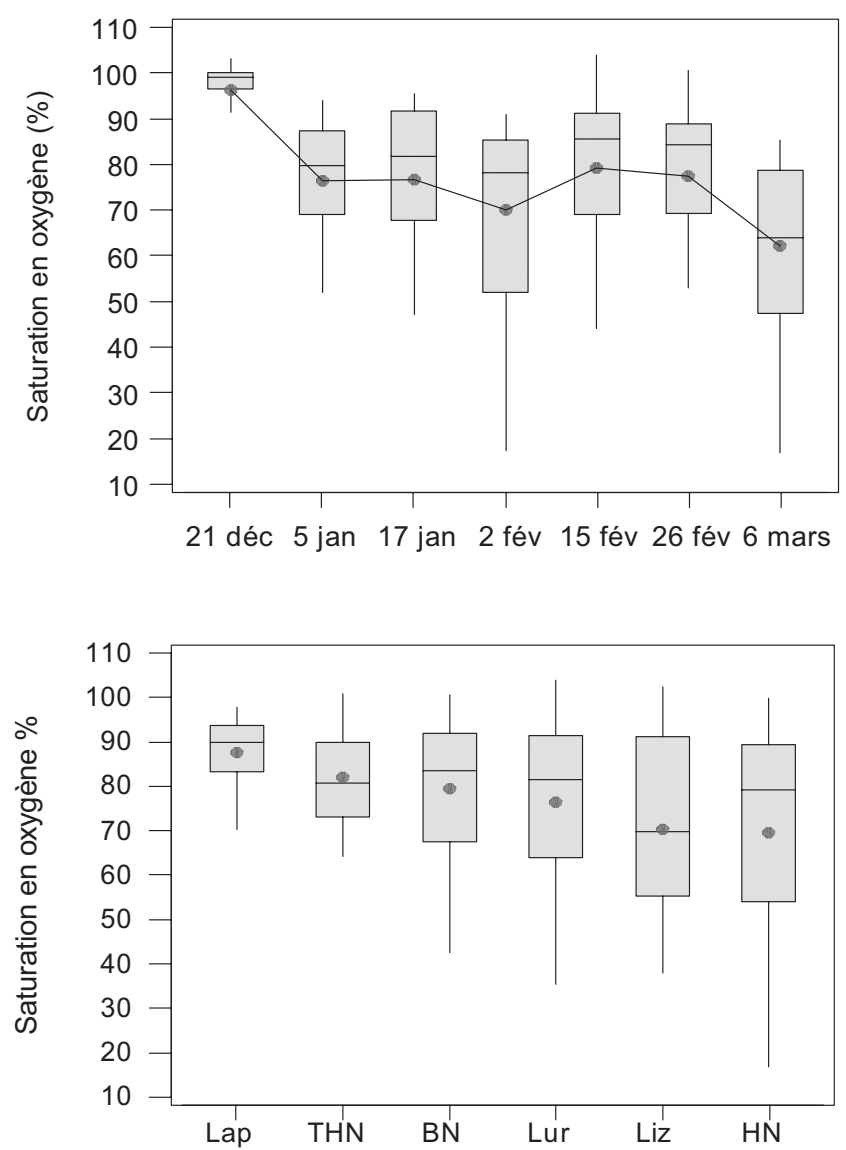

Figure 4

Boîtes à moustaches et valeurs moyennes (cercles) de la saturation en oxygène de l'eau interstitielle des frayères de la Nivelle ; (a) de décembre 2000 à mars 2001 ; (b) dans les différents tronçons de cours d'eau (BN : Basse Nivelle ; HN : Haute Nivelle ; THN : Très Haute Nivelle ; Lap : Lapitxuri ; Liz : Lizarrieta ; Lur : Lurgorriata).

\section{Figure 4}

Boxplots and mean values (circles) of oxygen saturations of interstitial water in the redds of the Nivelle; (a) from December 2000 to March 2001; (b) in the different reaches of rivers (BN: Lower Nivelle; HN: Upper Nivelle; THN: Very Upper Nivelle; Lap: Lapitxuri; Liz: Lizarrieta; Lur: Lurgorriata).

Le facteur tronçon révèle une influence significative sur la saturation en oxygène (analyse de variance à un facteur, $\mathrm{F}_{5,153}, \mathrm{p}<0,05$ ). Les valeurs les plus élevées sont enregistrées dans le Lapitxuri (87,6 \%) et en Très Haute Nivelle (82 \%), et les plus faibles dans le Lizarrieta $(70,2 \%)$ et en Haute Nivelle $(69,5 \%)$; la Basse Nivelle $(79,7 \%)$ et le Lurgorrieta $(76,3 \%)$ présentent des valeurs intermédiaires (Figure $4 b)$. Seules les valeurs extrêmes diffèrent (Lapitxuri contre Lizarrieta et Haute Nivelle ; tests de comparaison des moyennes de Tukey, $\mathrm{p}<0,05)$.

\section{Survie}

\section{Lot témoin}

En écloserie le taux de survie jusqu'à la résorption de la vésicule vitelline des alevins (770 dj), correspondant au stade de l'émergence en milieu naturel, s'élève à $88,5 \%$. II 
est très voisin de celui observé dans les mêmes conditions les années antérieures sur 14 pontes (88,9 \% ; BEALL et DUMAS, non publié).

\section{Survie en frayères}

Sur 3300 œufs introduits dans les capsules, 99,2\% sont récupérés à la fin des observations sous forme d'œufs morts $(68,7 \%)$, d'alevins vésiculés morts $(1,9 \%)$ et d'alevins vivants à la vésicule vitelline résorbée ou sur le point de l'être $(28,6 \%)$. La très petite fraction des effectifs non retrouvée s'ajoute aux mortalités des deux premiers groupes. II ressort que la majorité des mortalités intervient avant l'éclosion.

La position des capsules en amont ou en aval de la frayère n'influence pas le taux de survie $(29,4$ et $28 \%$, respectivement pour chaque groupe ; test de Wilcoxon, $N=19$; Figure $5 \mathrm{a})$. De même, la nature de la frayère n'a pas d'influence notable sur la survie $(23,9$ et $22,7 \%$ respectivement dans les frayères artificielles et naturelles ; test de Wilcoxon, $\mathrm{N}=5$; Figure $5 b)$.
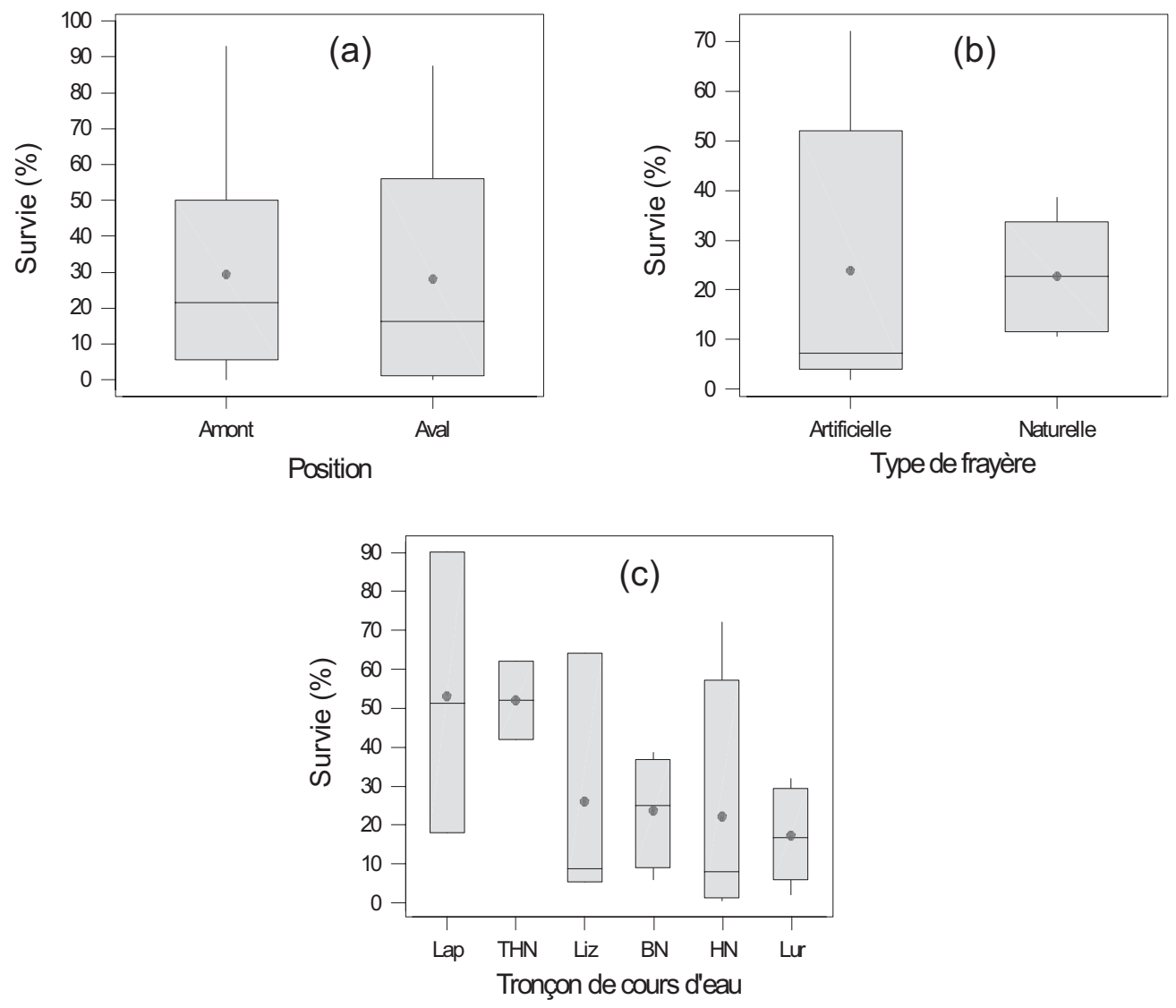

Figure 5

Boîtes à moustaches et valeurs moyennes (cercles) des taux de survie dans les frayères : (a) selon la position des groupes de capsules, (b) selon le type de frayère, (c) selon le tronçon de cours d'eau (BN : Basse Nivelle ; HN : Haute Nivelle ; THN : Très Haute Nivelle ; Lap : Lapitxuri ; Liz : Lizarrieta ; Lur : Lurgorriata).

\section{Figure 5}

Boxplots and mean values (circles) of survival rates in the redds: (a) depending on the position of capsule groups, (b) depending on the redd type, (c) depending on the reach of river (BN: Lower Nivelle; HN: Upper Nivelle; THN: Very Upper Nivelle; Lap: Lapitxuri; Liz: Lizarrieta; Lur: Lurgorriata). 
La moyenne de la survie au stade correspondant à celui de l'émergence sur l'ensemble du bassin de la Nivelle (28,6 \%) varie beaucoup d'un site l'autre (écart-type = 36,3\%). Elle est plus élevée dans les tronçons du Lapitxuri et de la Très Haute Nivelle $(52,7 \%$, Figure 5c) que dans les quatre autres tronçons (21,5\%; test de Mann-Whitney, $\mathrm{N}_{1}=5, \mathrm{~N}_{2}=17$, $p<0,05)$. Parmi ces derniers, seules deux frayères présentent des taux élevés, la frayère 4 située en amont du Lizarrieta (64 \%), et la frayère 15 de la Haute Nivelle (72\%), à proximité d'un tronçon où la survie est performante. Dans les trois tronçons du domaine accessible (Basse Nivelle, Haute Nivelle et Lurgorrieta) la survie est de 20,5\%.

\section{Relations entre survie et variables environnementales}

\section{Caractéristiques granulométriques et survie}

Aucune relation n'est mise en évidence entre la survie dans les frayères et les caractéristiques granulométriques du substrat d'incubation ( $\mathrm{Dg}, \mathrm{Fi}$, So et fractions de particules), aussi bien à l'état initial que final (corrélations de rang de Spearman, $N=17$ ).

\section{Oxygène de l'eau interstitielle et survie}

Des corrélations positives très fortes apparaissent entre taux de survie et taux de saturation en oxygène moyens et minima au cours de la période d'observation (corrélations de rang de Spearman, $\mathrm{N}_{1}=41, \mathrm{R}_{\mathrm{s} 1}=0,68, \mathrm{p}<0,001$, avec la saturation moyenne, $\mathrm{N}_{2}=41, \mathrm{R}_{\mathrm{s} 2}=0,67, \mathrm{p}<0,001$, avec la saturation minimum). La survie est d'autant meilleure que la concentration en oxygène est élevée (Figure 6). Au-dessous de

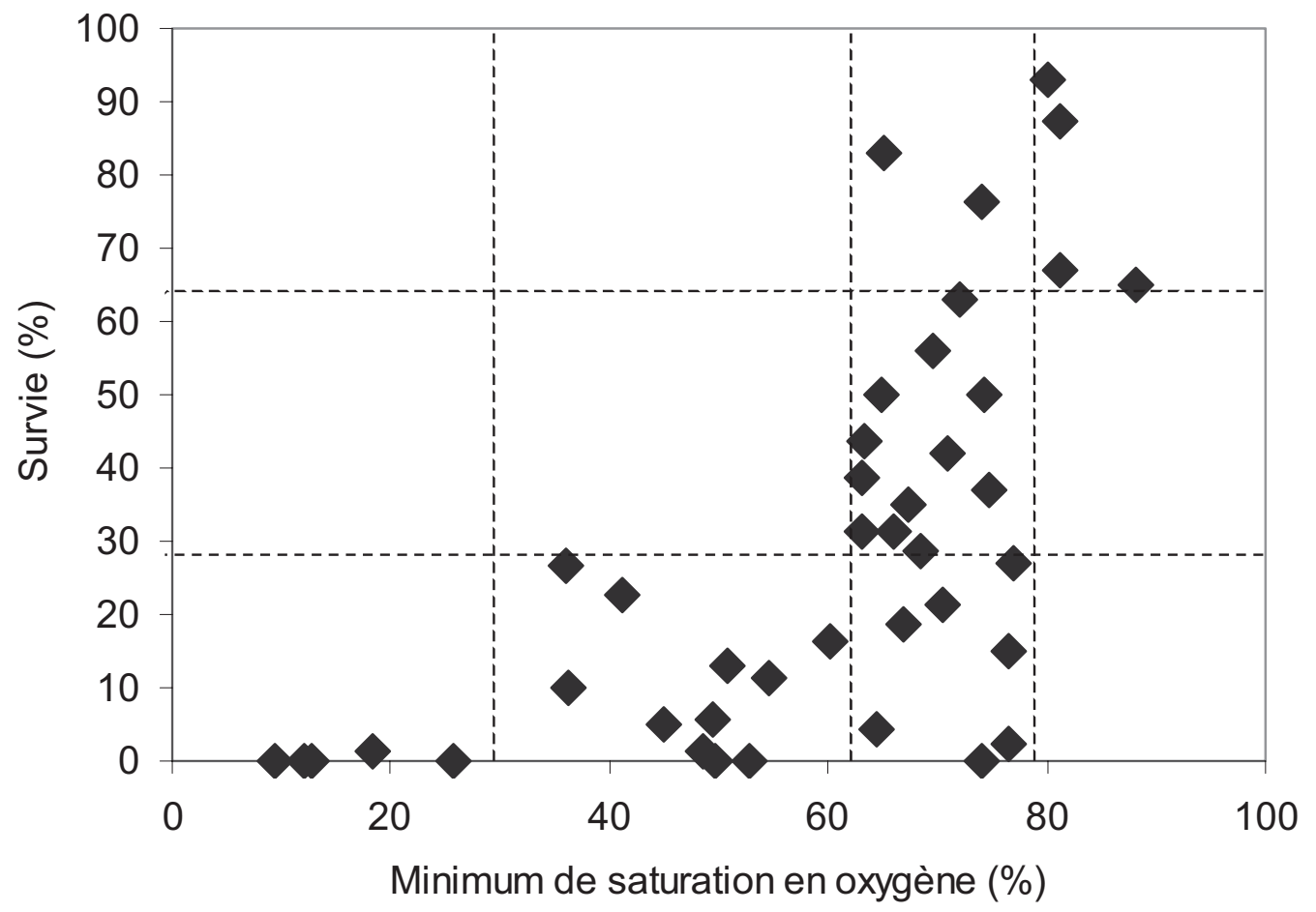

Figure 6

Relation entre la survie dans les groupes de capsules et la saturation minimum en oxygène de l'eau interstitielle des frayères de décembre 2000 à mars 2001

Figure 6

Relation between the survival rate in capsule groups and the minimum oxygen saturation rate of interstitial water from December 2000 to March 2001. 
$30 \%$ de saturation minimum $\left(3,3 \mathrm{mg}^{-1}\right)$ la survie est très faible ou nulle, entre cette limite et $62 \%\left(6,8 \mathrm{mg}^{-1}{ }^{-1}\right)$, elle est en moyenne de $10,2 \%$ (de 0 à $26,7 \%$ ). Entre $62 \%$ et $78 \%$ de saturation (respectivement 6,8 et $8,5 \mathrm{mg.l}^{-1}$ ), la survie moyenne s'élève à $35,9 \%$ mais demeure très variable (de 0 à $82,9 \%$ ). Au-dessus de $78 \%$ d'oxygène $\left(8,5 \mathrm{mg}^{\circ} \mathrm{l}^{-1}\right)$, elle atteint en moyenne 78,1 \% (de 65 à 92,9\%).

\section{DISCUSSION}

\section{Conditions environnementales}

\section{Morphologie des frayères}

La situation et les caractéristiques morphométriques des frayères artificielles sont en conformité avec les frayères naturelles observées dans la Nivelle (DUMAS et DAROLLES, 1999), ainsi que dans d'autres rivières (WHITE, 1942 ; STUART, 1953 ; BJORNN et REISER, 1991). Les nombres de Froude ( $\mathrm{Fr}=0,33$ et 0,34 en moyenne, respectivement pour les frayères artificielles et naturelles) sont aussi très voisins de ceux déjà observés dans la Nivelle (ESTOURNÈS, 1998 ; DUMAS et DAROLLES, 1999) et dans un affluent de la Dee écossaise (MOIR et al., 1998). Ces valeurs, très inférieures à 1, montrent que les frayères sont soumises à un écoulement peu perturbé. Cette similitude de caractéristiques entre frayères artificielles et naturelles se vérifie aux cinq sites où sont implantés les deux types de frayères où les teneurs en oxygène interstitiel et les taux de survie se révèlent très voisins. Ceci suggère une évolution comparable des caractéristiques structurales et de circulation d'eau bien que les modes de création de ces frayères soient différents.

\section{Températures et débits}

Les températures de l'eau, avec une moyenne près de $10^{\circ} \mathrm{C}$ et ne dépassant que rarement les $12^{\circ} \mathrm{C}$, demeurent compatibles avec les exigences de développement embryonnaire de l'espèce (STUART, 1953 ; BJORNN et REISER, 1991 ; CRISP, 1993).

On observe généralement que les épisodes de débits élevés, pendant lesquels le transport des particules fines augmente considérablement, entraînent le colmatage des frayères, voire leur destruction, hypothéquant ainsi indirectement ou directement la survie des œufs et des alevins (CHAPMAN, 1988 ; LISLE et LEWIS, 1992). Par forts débits, les vitesses élevées peuvent entraîner un décolmatage du substrat de surface qui rend temporairement les frayères plus perméables à l'eau de surface. Durant nos observations, le débit moyen demeure du même ordre de grandeur que ceux des années précédentes $\left(5,4\right.$ à $\left.6,7 \mathrm{~m}^{3} \cdot \mathrm{s}^{-1}\right)$. La fréquence de crues et leurs débits journaliers maxima observés, ne dépassant pas $16 \mathrm{~m}^{3} \cdot \mathrm{s}^{-1}$, sont normaux pour la saison dans ce cours d'eau (DUMAS et DAROLLES, 1999). Ils n'entraînent aucune destruction de frayères ou de leurs équipements. Ces crues provoquent un léger arasement du dôme des frayères, évalué à $1,7 \mathrm{~cm}$, ainsi qu'un comblement de la dépression amont; elles n'ont donc qu'un faible impact sur la modification de leur structure et de la qualité de leur substrat.

\section{Caractéristiques granulométriques}

Dans des travaux similaires on constate que les particules fines s'infiltrent dans le substrat des frayères principalement à l'occasion des crues (SEAR, 1993 ; ACORNLEY et SEAR, 1999 ; MASSA, 2000) et que sa qualité pour l'incubation se dégrade (PLATTS et al., 1979 ; CHAPMAN, 1988 ; PETERSON et QUINN, 1996a ; DUMAS et DAROLLES, 1999). De même, entre le début et la fin de notre expérience, le substrat d'incubation se colmate. Les frayères artificielles présentent des proportions de particules comparables à celles rapportées par CHAPMAN (1988). Le taux d'incorporation des fractions $<1 \mathrm{~mm}$ est faible (augmentation de $12 \%$ par rapport à leurs valeurs initiales), et la proportion d'éléments $>5 \mathrm{~mm}$ demeure sensiblement la même. Toutefois, des proportions de sables 
et de fines $<2 \mathrm{~mm}$ telles que celles observées (18\% ou plus) sont suffisantes pour réduire la disponibilité d'oxygène pour les œufs (GREIG et al., 2005) et induire des mortalités conséquentes. Ainsi, des proportions de 15 à $20 \%$ de ces éléments entraînent de fortes baisses de survie chez diverses espèces de salmonidés dans les frayères (MCNEIL, 1964 ; TAGART, 1976 ; HAUSLE et COBLE, 1976) ou en milieu contrôlé (PETERSON et METCALFE, 1981 ; LAPOINTE et al., 2004).

Parallèlement, Dg et Fi évoluent peu. II semble que les crues n'ont eu qu'une influence modérée sur le débit solide et notamment les plus grosses particules; les modifications du substrat lors de la création des frayères sont encore partiellement visibles en mars. Tout comme le remarquent BARRACOU (1994) ainsi que DUMAS et DAROLLES (1999), la grande variabilité dans les échantillons de substrat, que ce soit en début ou en fin d'incubation, ne permet pas de distinguer un gradient aval-amont d'accumulation des fines et de dégradation prononcée du milieu d'incubation.

\section{Qualité de l'eau interstitielle}

Bien que l'eau de surface soit constamment saturée en oxygène, le taux de saturation intra-frayère diminue en moyenne de $35 \%$ au cours de l'incubation. Cette évolution est normale et résulte du changement de qualité du substrat et d'autres facteurs du milieu interstitiel. L'activité biochimique (respiration des embryons dans le chorion ou vésiculés, décomposition de ceux qui sont morts, activité bactérienne) tend à faire chuter le taux d'oxygène (VAUX, 1962). La morphologie particulière de la frayère (une dépression suivie d'un dôme), qui favorise la pénétration de l'eau dans le substrat (VAUX, 1962), est progressivement nivelée par les crues, ce qui limite ce processus. De plus, les sédiments fins qui pénètrent dans le substrat à ces occasions réduisent la circulation interstitielle de l'eau et les apports d'oxygène en provenance de l'eau de surface (VAUX, 1962 ; BESCHTA et JACKSON, 1979 ; SCRIVENER et BROWNLEE, 1989).

Suite aux différents épisodes de crues, les teneurs moyennes en oxygène dissous chutent, mais demeurent le plus souvent au-dessus de $8 \mathrm{mg}^{-\mathrm{I}^{-1}}$ (soit $75 \%$ de saturation) à l'exception des périodes d'éclosion et d'émergence. Elles sont suffisantes pour permettre une survie satisfaisante des œufs et des alevins (PHILLIPS et CAMPBELL, 1962 ; BJORNN et REISER, 1991 ; CRISP, 1993). Au moment des éclosions, peu après une période de crues, la concentration moyenne s'abaisse à $7,5 \mathrm{mg}^{-l^{-1}}$ ( $69 \%$ de saturation) avec des minima au-dessous de 5,8 mg.l-1 (53\% de saturation). Cette dernière concentration correspond au seuil minimum toléré par l'espèce à ce stade de développement (WICKETT, 1954 ; DAVIS, 1975). En effet, c'est peu avant et au cours de l'éclosion que les besoins en oxygène de l'embryon sont les plus forts; ce dernier s'agite pour sortir du chorion, ce qui accroît sa consommation d'oxygène alors que la surface d'échanges gazeux reste limitée à cette enveloppe (ROMBOUGH, 1988 ; MASSA, 2000).

Bien que les teneurs moyennes et minima en oxygène dépendent du degré de colmatage du substrat (RUBIN, 1995 ; RUBIN et GLIMSÄTER, 1996), aucune relation n'est mise en évidence avec les différentes caractéristiques granulométriques en début et fin d'expérience. PETERSON et QUINN (1996b), ESTOURNÈS (1998) ainsi qu'INGENDAHL (2001) avaient déjà observé cette absence de corrélation entre ces deux variables pour les frayères de salmonidés. Or, la concentration en oxygène de l'eau hyporhéique et des frayères de certains cours d'eau peut dépendre très majoritairement du flux en provenance de l'eau de surface et donc de la composition granulométrique du substrat des frayères qui en limite la circulation. Dans d'autres systèmes, notamment dans la partie aval des cours d'eau et en plaine, le flux d'eau interstitielle peut provenir d'un mélange d'eau de nappe phréatique ou profonde pauvre en oxygène (HANSEN, 1995 ; SOULSBY et al., 2000) et d'eau de surface en proportions variables (MALCOLM 
et al., 2003). II est possible que ce phénomène intervienne dans certaines frayères du bassin de la Nivelle où le lit majeur est large et constitué d'alluvions et où il existe des échanges entre la nappe alluviale et le cours d'eau (OLAïZOLA, 2001). Dans ce cas, la qualité de l'eau interstitielle est presque indépendante de la taille des particules du substrat, du matériel biologique en décomposition et de la demande biologique en oxygène (SOWDEN et POWER, 1985).

\section{Survie à l'émergence et relations avec les facteurs environnementaux}

Une fois la vésicule vitelline résorbée, stade homologue à celui des alevins émergents, la survie pour l'ensemble des frayères (près de $29 \%$ ), se situe dans la fourchette haute des valeurs avancées pour le saumon Atlantique dans différentes rivières européennes et américaines (2 \% à $35 \%$ ) selon BLEY et MORING (1988), MACKENZIE et MORING (1988), PAUWELS et HAINES (1994), RUBIN (1995), O'CONNOR et ANDREW (1998). Elle est proche de celle observée par ESTOURNÈS (1998) dans ce même ensemble de cours d'eau (25\%). Toutefois cette dernière estimation est obtenue avec des boites d'incubation qui tendent à surestimer la survie, comparativement aux capsules utilisées dans cette expérience, par augmentation de la perméabilité du substrat d'incubation. En effet, une perte partielle des éléments fins est observée sur le gravier de remplissage des boîtes pris dans les frayères réceptrices, ainsi que sur le substrat environnant lors de l'enfouissement de celles-ci (DUMAS et MARTY, 2006).

Les zones d'amont, où la qualité de l'eau est peu dégradée par comparaison aux zones de l'aval (DUMAS et HAURY, 1995) sont celles où la survie sous graviers est la meilleure ; avec $53 \%$ de survie, la Très Haute Nivelle française et le Lapitxuri se révèlent 2,5 fois plus efficaces pour le succès de la reproduction que les autres tronçons. Dans le domaine accessible au saumon le taux de survie est de 20,5\%, alors qu'il était précédemment estimé à $12 \%$ dans des frayères naturelles avec des filets-capes, structures lenticulaires filtrantes pourvues d'un piège à alevin qui recouvrent la surface de chaque frayère ainsi équipée (DUMAS et DAROLLES, 1999), et à $31 \%$ avec des boîtes d'incubation (ESTOURNÈS, 1998).

Plus des $2 / 3$ des mortalités ont lieu avant l'éclosion, période au cours de laquelle interviennent la majorité des crues. Entre ce stade et l'émergence des alevins, le débit ne subit pas de variations majeures et les mortalités sont faibles (6\% des embryons éclos). Ceci confirme que les événements hydrologiques ont une influence négative sur la survie, ce qui est déjà signalé par CHAPMAN (1988) et pris en compte par LISLE et LEWIS (1992) et GUERRIN et DUMAS (2001). RUBIN et GLIMSÄTER (1996), puis MASSA (2000) observent aussi, pour la première partie d'incubation une fragilité des embryons de truite commune, Salmo trutta, dont les mortalités sont fonction des teneurs en oxygène, en ammonium et en nitrites. Ces deux derniers éléments, mesurés en faibles quantités par ESTOURNES (1998), n'influenceraient pas les taux de survie des embryons de saumons dans ce bassin versant.

Les caractéristiques du substrat agissent avec certitude sur la survie embryonnaire des salmonidés (CHAPMAN, 1988), or aucune corrélation entre ces caractéristiques et la survie n'est mise en évidence dans le cadre de cette expérience, ce qui est la conséquence directe de l'absence de lien entre caractéristiques du substrat et oxygène dissous. Ainsi dans les conditions de cette étude, les indices granulométriques établis ne semblent pas être des paramètres permettant d'interpréter la survie.

Comme dans notre expérience, MALCOLM et al. (2003) avec des frayères artificielles de truite commune dans un cours d'eau de bassin versant agricole dégradé notent des taux de survie positivement corrélés à la concentration moyenne d'oxygène de la période d'observation. Ils ne rapportent des survies $>35-40 \%$ qu'avec des concentrations en oxygène $>8,5 \mathrm{mg} . \mathrm{l}^{-1}$, soit $75-80 \%$ de saturation pour la gamme de températures 
observées dans notre étude. Dans le cas du bassin de la Nivelle, la saturation en oxygène à proximité des œufs a une influence significative sur la survie et les minima sont limitants. Cependant, elle n'explique pas complètement celle-ci, notamment lorsque ces minima se situent entre 62 et $78 \%$ alors que la survie varie beaucoup. Cette grande dispersion de survie pour des valeurs proches de saturation pourraient dépendre de la variabilité à échelle de temps réduite de l'oxygène dissous de l'eau interstitielle (MALCOLM et al., 2003) ; l'eau peu oxygénée de la nappe peut s'exfiltrer dans les frayères lors des décrues, entraîner des mortalités et ne pas être détectée, faute d'un pas de temps adéquat d'échantillonnage de l'eau.

\section{CONCLUSION}

Les capsules d'incubation, comparées aux méthodes jusque là utilisées, filetscapes sur frayère pour piéger les alevins émergents et boîtes d'incubation-émergence enfouies dans le substrat, confirment les avantages déjà soulignés par DUMAS et MARTY (2006). Ce dispositif, d'un plus faible volume que celui utilisé par SCRIVENER (1988) est facile à mettre en œuvre. II respecte mieux la structure du substrat que les boîtes, de plus grand volume, dont le remplissage et l'enfouissement provoquent une perte de fines améliorant ainsi le milieu d'incubation, ce qui avantage la survie. II est aussi plus efficace que les filets-capes dont la mise en place déstructure le gravier au travers duquel des alevins peuvent s'échapper, occasionnant une survie apparente moindre. N'offrant qu'une très faible prise au courant, les capsules sont peu vulnérables aux crues. Elles procurent ainsi des valeurs de survie sur la totalité de la période de développement embryonnaire, plus réalistes, intermédiaires entre celles des deux autres méthodes (DUMAS et MARTY, 2006).

Cette expérience met en évidence une forte variabilité de la survie embryonnaire du saumon Atlantique d'un site de frai à l'autre dans le bassin de la Nivelle. II ressort que cette survie, pénalisée par les épisodes de crues, dépend moins directement de la composition et de la structure granulométrique du substrat de frai que des conditions physicochimiques aux alentours immédiats des œufs et des alevins, révélées par l'oxygène dissous de l'eau interstitielle. La saturation en oxygène de celle-ci est susceptible de varier au gré des intrusions d'eau de la nappe alluviale et de l'eau de surface pendant et après les crues. De façon à mieux apprécier ces variations, il conviendrait de contrôler la qualité de l'eau interstitielle à des pas de temps plus fréquents que ceux adoptés ici, de l'ordre de 3 à 7 jours en période de débits fluctuants.

Les zones amont du bassin versant de la Nivelle, plus particulièrement de la Très Haute Nivelle française et de son affluent, le Lapitxuri, où la survie est 2,5 fois plus élevée que dans les tronçons actuellement accessibles aux géniteurs, se révèlent de bien meilleure qualité pour l'efficacité du frai. II serait important pour la population de saumons de ce cours d'eau, en limite Sud de l'aire de répartition de l'espèce, d'ouvrir aux reproducteurs l'accès à ces zones de frai du haut bassin français et espagnol, qui, dans un contexte de changement climatique, présentent des conditions, et en particulier de températures, plus favorables et un potentiel de production de juvéniles plus conséquent que les zone de l'aval.

\section{REMERCIEMENTS}

Nous tenons à remercier la DIREN Aquitaine pour sa contribution financière, I'association MIGRADOUR pour le piégeage des géniteurs, L'AAPPMA de la Nivelle et la garderie du Conseil Supérieur de la Pêche pour les opérations de repérage et de surveillance des frayères. Nous remercions également les deux relecteurs anonymes pour leurs commentaires très utiles et les améliorations apportées au manuscrit. Nous 
sommes très redevables envers les personnes ayant participé à la mise en place et au bon déroulement de l'expérience, tout particulièrement E. HUCHET, S. GLISE, J. RIVES et J.C. VIGNES.

\section{BIBLIOGRAPHIE}

ACORNLEY R.M., SEAR D.A., 1999. Sediment transport and siltation of brown trout (Salmo trutta L.) spawning gravels in chalk steams, Hydrological Processes, 13, 447-458.

BARRACOU D., 1994. Étude de la qualité des frayères à saumon des bassins des Gaves et des Nives, Conseil Supérieur de la Pêche, Délégation Régionale Midi-Pyrénées Aquitaine, antenne de Pau, $39 \mathrm{p}$.

BESCHTA R.L., JACKSON W.L., 1979. The intrusion of fine sediments into a stable gravel bed, Journal of the Fisheries Research Board of Canada, 36, 204-210.

BJORNNT.C., REISER D.W., 1991. Habitat requirements of salmonids in streams, American Fisheries Society Special Publication, 19, 83-138.

BLEY P.W., MORING J.R., 1988. Freshwater and ocean survival of Atlantic salmon and steelhead: A synopsis, U.S. Fish and Wildlife Service, Biological Report, 88 (9), $22 \mathrm{p}$.

BURKHALTER D.E., KAYA C.M., 1977. Effects of prolonged exposure to ammonia on fertilised eggs and sac-fry of rainbow trout (Salmo gairdneri). Transactions of the American Fisheries Society, 106, 470-475.

CHAPMAN D.W., 1988. Critical review of variables used to define effects of fines in redds of large salmonids, Transactions of the American Fisheries Society, 117, 1-21.

CRISP D.T., 1993. The environmental requirements of salmon and trout in fresh water, Freshwater Forum, 3, 176-202.

CRISP D.T., 1996. Environmental requirements of common riverine European salmonid fish species in fresh water with particular reference to physical and chemical aspects, Hydrobiologia, 323, 201-221.

DAVIS J.C., 1975. Minimal dissolved oxygen requirements of aquatic life with emphasis on Canadian species : a review, Journal of the Fisheries Research Board of Canada, 32, 2295-2330.

DUMAS J., HAURY J., 1995. Une rivière du piémont pyrénéen : la Nivelle (Pays Basque), Acta Biologica Montana, 11, 113-146.

DUMAS J., DAROLLES V., 1999. Caractéristiques environnementales et survie embryolarvaire du Saumon atlantique, Salmo salar L., dans un cours d'eau du piémont pyrénéen, la Nivelle (France), Cybium, 23 (1) suppl., 29-44.

DUMAS J., MARTY S., 2006. A new method to evaluate egg-to-fry survival in salmonids, trials with Atlantic salmon, Journal of Fish Biology, 68, 284-304.

DUMAS J., BASSENAVE J.G., BARRIERE L., GLISE S., Sous presse. Effects of fish farm effluents on egg-to-fry developpement and survival of brown trout in redds, Journal of Fish Biology, 70.

DUMAS J., PROUZET P., 2003. Variability of demographic parameters and population dynamics of Atlantic salmon (Salmo salar L.) in a southwest French river, ICES Journal of Marine Science, 60, 356-370.

ESTOURNES G., 1998. Étude de la survie embryolarvaire du Saumon atlantique (Salmo salar L.) dans la Nivelle. Influence de la qualité des frayères et de l'eau interstitielle, Diplôme d'Étude Supérieure, Université Bordeaux I, 33 p. 
GREIG S.M., SEAR D.A., SMALLMAN D., CARLING P.A., 2005. Impact of clay particles on the cutaneous exchange of oxygen across the chorion of Atlantic salmon eggs, Journal of Fish Biology, 66, 1681-1691.

GUERRIN F., DUMAS J., 2001. Knowledge representation and qualitative simulation of salmon redd functioning. Part II : Qualitative model of redd, Biosystems, 59, 85108.

HANSEN E.A., 1995. Some effects of groundwater on brown trout redds, Trasactions of the American Fisheries Society, 1, 100-110.

HAUSLE D.A., COBLE D.W., 1976. Influence of sand in redds on survival and emergence of brook trout (Salvelinus fontinalis), Transactions of the American Fisheries Society, $105,57-63$.

HÉLAND M., DUMAS J., 1994. Écologie et comportement des juvéniles. In : GUEGUEN J.C., PROUZET P. (Eds.), Le Saumon atlantique, 29-46, I.F.R.E.M.E.R, Plouzané.

INGENDAHL D., 2001. Dissolved oxygen concentration and emergence of sea trout fry from natural redds in tributaries of the River Rhine, Journal of Fish Biology, 58, 325-341.

LAPOINTE M.F., BERGERON N.E., BERUBE F., POULIOT M.A., JOHNSTON P., 2004. Interactive effects of substrate sand and silt contents, redd-scale hydaulic gradients, and interstitial velocities on egg-to-emergence survival of Atlantic salmon (Salmo salar), Canadian Journal of Fisheries and Aquatic Sciences, 61, 2271-2277.

LISLE T.E., LEWIS J., 1992. Effects of sediment transport on survival of salmonid embryos in a natural stream : a simulation approach, Canadian Journal of Fisheries and Aquatic Sciences, 49, 2337-2344.

LOTSPEICH F.B., EVEREST F.H., 1981. A new method for reporting and interpreting textural composition of spawning gravel, U.S. Forest Service, Research Note PNW$369,11 \mathrm{p}$.

MACKENZIE C., MORING J.R., 1988. Estimating survival of Atlantic salmon during the intra-gravel period, North American Journal of Fisheries Management, 8, 45-49.

McNEIL W.J., 1964. Redd superimposition and egg capacity of pink salmon spawning beds, Journal of the Fisheries Research Board of Canada, 21, 1385-1396.

MALCOM I.A., YOUNGSON A.F., SOULSBY C., 2003. Survival of salmonid eggs in a degraded gravel-bed stream : effects of groundwater-surface water interactions, River Research and Applications, 19, 303-316.

MASSA F., 2000. Sédiments, physico-chimie du compartiment interstitiel et développement embryolarvaire de la truite commune (Salmo trutta): Étude en milieu naturel anthropisé et en conditions contrôlées, Thèse de Doctorat, Institut National Agronomique, Paris Grignon, $178 \mathrm{p}$.

MOIR H.J., SOULSBY C., YOUNGSON A., 1998. Hydraulic and sedimentary characteristics of habitat utilized by Atlantic salmon for spawning in the Girnock Burn, Scotland, Fisheries Management and Ecology, 5, 241-254.

O'CONNOR W.C.K., ANDREWT.E., 1998. The effects of siltation on Atlantic salmon, Salmo salar L., embryos in the River Bush, Fisheries Management and Ecoogy, 5, 393401.

OLAïZOLA M., 2001. Variabilité de la survie embryolarvaire du saumon Atlantique (Salmo salar L.) dans la Nivelle. Influence des facteurs environnementaux, Diplôme d'Études Supérieures, Université Bordeaux I, 45 p. 
PAUWELS S.J., HAINES T.A., 1994. Survival, hatching, and emergence success of Atlantic Salmon eggs planted in three Maine streams, North American Journal of Fisheries Management, 14, 125-130.

PETERSON R.H., METCALFE J.L., 1981. Émergence of Atlantic salmon fry from gravels of varying composition : a laboratory study, Canadian Technical Report of Fisheries and Aquatic Sciences, 1020, iii + 15 p.

PETERSON N.P., QUINN T.P., 1996a. Persistence of egg pocket architecture in redds of chum salmon, Oncorhynchus keta, Environmental Biology of Fishes, 46, 243-253.

PETERSON N.P., QUINN T.P., 1996b. Spatial and temporal variation of dissolved oxygen in natural egg pockets of chum salmon in Kennedy Creek, Washington, Journal of Fish Biology, 48, 131-143.

PHILLIPS R.W., CAMPBELL H.J., 1962. The embryonic survival of coho salmon and steelhead trout as influenced by some environmental conditions in gravel beds, Pacific Marine Fisheries Commission Annual Report, 14, 60-73.

PLATTS W.S., SHIRAZI M.A., LEWIS D.H., 1979. Sediment particle sizes used by salmon for spawning with methods for evaluation. U.S. Environmental Protection Agency, Ecological Research Series, EPA-600/3-79-043, 32 p.

ROMBOUGH P.J., 1988. Respiratory gas exchange, aerobic metabolism, and effects of hypoxia during early life. In : HOAR W.S., RANDALL D.J. (Eds), Fish Physiology, 11A., 59-161, Academic Press, San Diego.

RUBIN J.-F., 1995. Estimating the success of natural spawning of salmonids in streams, Journal of Fish Biology, 46, 603-622.

RUBIN J.-F., 1998. Survival and emergence pattern of sea trout fry in substrata of different compositions. Journal of Fish Biology, 53, 84-92.

RUBIN J.-F., GLIMSÄTER C., 1996. Egg-to-fry survival of the sea trout in some streams of Gotland. Journal of Fish Biology, 48, 585-606.

SCRIVENER J.C., 1988. Two devices to assess incubation survival and emergence of salmonid fry in an estuary streambed. North American Journal of Fisheries Management, 8, 248-258.

SCRIVENER J.C., BROWNLEE M.J., 1989. Effects of harvesting on spawning gravel and incubation survival of chum (Oncorhynchus keta) and coho salmon (O. kisutch) in Carnation Creek, British Columbia, Canadian Journal of Fisheries and Aquatic Sciences, 46, 681-696.

SEAR D.A., 1993. Fine sediment infiltration into gravel spawning beds within a regulated river experiencing floods : ecological implication for salmonids, Regulated Rivers : Research and Management, 8, 373-390.

SOULSBY C., MALCOLM R., HELLIWELL R.C., FERRIER R.C., JENKINS A., 2000. Isotope hydrology of the Allt a'Mharcaidh catchment, Hydrological Processes, 14, 747-762.

SOWDEN T.K., POWER G., 1985. Prediction of rainbow trout embryo survival in relation to groundwater seepage and particle size of spawning substrates, Transactions of the American Fisheries Society, 114, 804-812.

STUART T.A., 1953. Spawning migration, reproduction and young stages of Loch trout (Salmo trutta L.), Scottish Home Department, Freshwater and Salmon Fisheries Research, 5, 1-39.

TAGART J.V., 1976. The survival from egg deposition to emergence of coho salmon in Clearwater River, Jefferson County, Washington, Master's thesis. University of Washington, Seattle, $101 \mathrm{p}$. 
VAUX W.G., 1962. Interchange of steam and intra-gravel water in a salmon spawning riffle, U.S. Fish and Wildlife Service, Special Scientific Report, Fisheries, 405.

WHITE H.C., 1942. Atlantic Salmon redds and artificial spawning beds, Journal of the Fisheries Research Board of Canada, 6, 37-44.

WICKETT W.P., 1954. The oxygen supply to salmon eggs in spawning beds Journal of the Fisheries Research Board of Canada, 11, 933-953. 